\title{
Enfoque Contextualizado para el Desarrollo de la Investigación Artística Musical
}

\section{Contextualized Approach for the Development of Musical Artistic Research}

\author{
Iris Liliana Vásquez-Alburqueque ${ }^{1}$ y Wilson Hugo Chunga-Amaya
}

\section{EDICIÓN. Resiliencia}

Recibido: 24/febrero/2021

Aceptado: 3/junio/2021

P Publicado: 29/noviembre/2021

\section{Páginas: 5-11}

\&aís

${ }^{1}$ Perú

${ }^{2}$ Perú

\section{IIIII Institución}

${ }^{1}$ Universidad Nacional de Frontera

${ }^{2}$ Universidad Antenor Orrego

\section{Correo Eletrónico}

1irli333@hotmail.com

²wilsoniuris_21@hotmail.com

\section{(D) ORCID}

${ }^{1}$ https://orcid.org/0000-0001-9831-3213 ${ }^{2} \mathrm{https}: / /$ orcid.org/0000-0003-4600-4886

\section{Citar así: C APA / IEEE}

Vásquez-Alburqueque, I. \& ChungaAmaya, W. (2021). Enfoque Contextualizado para el Desarrollo de la Investigación Artística Musical. Revista Tecnológica-Educativa Docentes 2.0, 1(1), 5-11

https://doi.org/10.37843/rted.v1i1.213

I. Vásquez-Alburqueque y W. ChungaAmaya, "Enfoque Contextualizado para el Desarrollo de la Investigación Artística Musical", RTED, vol. 1, n. ${ }^{\circ}$ 1, pp. 5-11, nov. 2021.

\section{Resumen}

En los últimos 20 años, poco se ha impulsado el crecimiento de la investigación artística musical. Para desarrollarla este tipo de investigación se necesitó que el docente adaptará la programación curricular y en la Universidad Nacional de Frontera los planes curriculares que no se han contextualizados por diversas razones académicas. El objetivo de esta investigación fue proponer un programa curricular contextualizado que desarrolle la investigación artística musical en estudiantes de administración, hotelera y de turismo. En el presente estudio surgieron 4 preguntas de investigación: ¿cómo identificar el desarrollo de investigación artística?, ¿qué fundamentos teóricos se debe considerar para caracterizar el programa?, ¿cómo validarlo? y ¿qué aspectos considerar?; que dieron origen a una propuesta metodología cuantitativa de tipo no experimental, propositiva. Se elaboraron tres cuestionarios, estos fueron aplicados a 27 estudiantes, 6 jerárquicos, 2 docentes. En esta investigación se aplicaron tres cuestionarios de 9 preguntas cada uno: a los estudiantes, jerárquicos y docentes quienes enseñaron el curso de arte en este semestre. Se concluyó que la investigación artística musical no se fomenta porque los docentes asignados a enseñar el curso de arte, sólo el 50\% son de la especialidad, los programas curriculares y sílabos no están contextualizados para el desarrollo de la investigación artística musical y no se promueven proyectos formativos del área en mención.

Palabras clave: Programación, contextualizado, investigación, artística, musical.

\section{Abstract}

In the last 20 years, little has fueled the growth of musical, artistic research. To develop this type of research, the teacher needed to adapt the curricular programming and the curricular plans that have not been contextualized for various academic reasons at the National University of Frontera. This research aimed to propose a contextualized curricular program that develops musical, artistic research in administration, hotel, and tourism students. In this study, four research questions arose: how to identify the development of artistic research? What theoretical foundations should be considered to characterize the program? How to validate it? And what aspects to consider? which gave rise to a proposed non-experimental, propositional quantitative methodology. Three questionnaires were developed; these were applied to 27 students, six hierarchical, two teachers. In this research, three questionnaires of 9 questions were used: the students, hierarchies, and teachers who taught the art course this semester. It concluded that musical, artistic research is not promoted because of the teachers assigned to teach the art course; only $50 \%$ are from the specialty. The curricular programs and syllables are not contextualized for the development of musical, artistic research. They have not promoted training projects in the area in question.

Keywords: Programming, contextualized, research, artistic, musical. 


\section{Introducción}

En los últimos 20 años, poco se ha impulsado el crecimiento de la investigación artística musical. De acuerdo con lo sustentado por López-Cano "Requiere de una sistematización metodológica particular de experiencias adquiridas en el entorno del estudiante, las cuales pueden recogerse a través de trabajos etnográficos" (2013, p. 86). Sin embargo, Vegas sustento "que se necesita crear un gabinete especializado, realizar trabajos de campo para crear archivos sonoros y fotográficos, coleccionar instrumentos musicales y crear un instituto de musicología" (1931, p.12). Por ello, diversos investigadores concuerdan que los estudiantes no desarrollan la investigación artística musical, porque ésta no se le reconoce como un lenguaje particular.

Por otro lado, Montero cree firmemente que es "imprescindible abrir espacios para el diálogo con las sociedades de compositores a través de foros, cursos, conferencias, actos especiales y así investigar permanentemente su obra" (2001, p.01). Asimismo, cuando se atribuye un objeto de estudios a una sola disciplina, se configura una dificultad para desarrollar investigación musical (OtaolaGonzález, 2015, p. 90). Evidentemente, por eso hoy en día los proyectos de investigación artística musical siguen teniendo una presencia débil en las universidades, a causa de la descontextualización de los programas curriculares que no permiten generar nuevo conocimiento que conecte con la realidad del estudiante y que los motive para investigar por ejemplo la música de su pueblo aprendiendo desde lo básico que se requiere.

En ese sentido, nuestro punto focal fueron los estudiantes del II ciclo del curso de arte de la Universidad Nacional de Frontera del Perú, donde se ha identificado que no han tenido experiencias previas de investigación sobre patrimonios musicales de su región, desconocen el proceso metodológico y las técnicas de la investigación artística musical. Este problema es importante solucionarlo porque permite a los estudiantes entrenarse en el ejercicio metodológico de la investigación y aprender aplicar técnicas basadas en el marco metodológico-teórico, que a su vez están sustentadas en el marco normativo de la ley universitaria $\mathrm{N}^{\circ} 30220$ que a su letra en su artículo 48 refiere: "la investigación constituye una función esencial y obligatoria de la universidad, que la fomenta y realiza, respondiendo a través de la producción del conocimiento" (Ley Universitaria 30220, 2014, p.25)

Ante esta problemática, el objetivo principal fue proponer un programa curricular basado en un enfoque contextualizado para desarrollar investigación artística en los estudiantes del II ciclo del curso arte de Facultad de Administración, Hotelera y de Turismo de Universidad Nacional de Frontera de Sullana - Piura - 2020. Por esta razón, se elaboró un programa curricular para ser incorporado en el silabo en 8 semanas (una unidad), el cual contiene 8 sesiones de clases, material de trabajo, rubricas de evaluación, este material didáctico creativo permitirá mejorar su nivel de conocimiento de los estudiantes sobre las técnicas de investigación artística.

\section{Metodología}

El diseño metodológico responde al enfoque cuantitativo de tipo no experimental, de estudio propositivo, porque el investigador no realizó un proceso de aplicación de la propuesta curricular con enfoque contextualizado, sólo aplicó tres instrumentos de investigación a estudiantes del curso de arte del II ciclo, docentes asignados a enseñar el curso de arte y jerárquicos de la facultad de Administración, Hotelera y de Turismo para diagnosticar el problema en toda su magnitud. Posterior a ello, con dichos resultados permitieron elaborar y validar la propuesta de programación curricular basado en un enfoque contextualizado a través de un juicio de expertos conformados por un metodólogo, un especialista. De acuerdo con el autor Hernández-Sampieri, et al. (2014), el diseño de investigación de tipo propositivo fue el ilustrado en la Figura 1: 


\section{Figura 1}

Diseño Metodológico

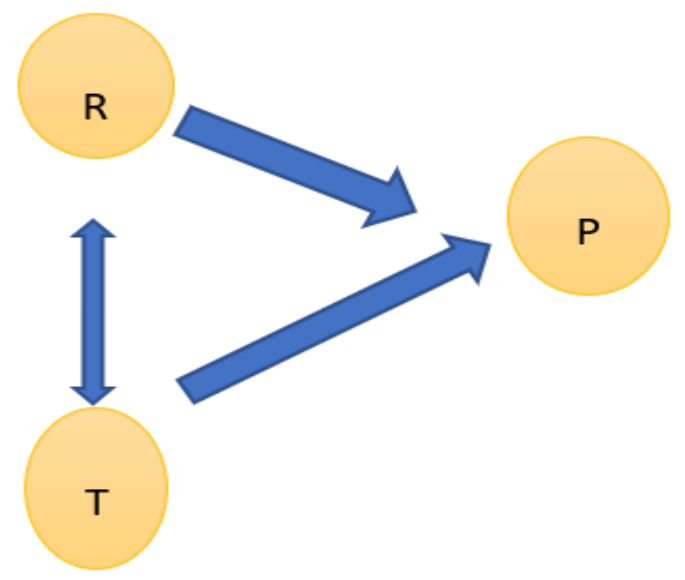

Nota. Donde, R: Diagnóstico de realidad, T: Estudios teóricos y P: Propuesta, elaborado por Vásquez \& Chunga (2020).

Por lo tanto, surgió la siguiente hipótesis si se proponer un programa curricular basado en un enfoque contextualizado, entonces si contribuirá a desarrollar investigación artística musical en los estudiantes del II ciclo del curso arte de Facultad de Administración, Hotelera y de Turismo de Universidad Nacional de Frontera de Sullana - Piura -2020.

En el caso de las variables utilizadas fueron las siguientes: independiente (programa curricular) y dependiente (investigación artística musical). Asimismo, la muestra usada consideró el criterio de inclusión, porque "el grupo era un conjunto de seres que poseían una característica en común" (Castillo \& Herrera, 2017), ellos fueron estudiantes del 2 do ciclo de facultad de administración, hotelera y de turismo de Universidad Nacional de Frontera de Sullana, grupo 1 quienes llevaron el curso de arte en el semestre 2020.

Por la manera de cómo se seleccionó a los estudiantes, jerárquicos, docentes para almacenar la información, fue un muestreo no probabilístico intencional, conocido también como muestra dirigida por seleccionar a los encuestados que le permitan alcanzar los objetivos de investigación. Además, para construir la estructura teórica se utilizó el método teórico donde revisó, seleccionó, y diseñó un marco teórico-metodológico para orientar las dimensiones e indicadores de variable dependiente, variable independiente, con la finalidad de estructurar los instrumentos para recolectar los datos. Del mismo modo, se revisaron diversas teorías para proponer una propuesta curricular basado en un enfoque contextualizado.

Finalmente, luego de aplicar los tres instrumentos se presentan los resultados en tablas, cuadros estadísticos con sus respectivos gráficos, resumen los estadígrafos donde se usó formulas estadísticas del programa de Excel, formatos de Google Form. Para la frecuencia porcentual se utilizó estadística básica, método que "consiste en clasificar, codificar, procesar e interpretar información obtenida durante recolección de los datos y su finalidad es llegar a conclusiones específicas del evento de estudios, dar respuesta a las preguntas de investigación" (Hurtado, 2000). A continuación, se presenta la Tabla 1 del objeto de estudios:

\section{Tabla 1}

Estudiantes del 2do Ciclo de la Facultad de Administración, Hotelera y de Turismo, Grupo 1

\begin{tabular}{lllll}
\hline \multirow{2}{*}{ ICLOS } & \multicolumn{2}{l}{ CARACTERÍSTICAS } & F & $\mathbf{h}^{\mathbf{1}}$ \\
\cline { 2 - 5 } & VARONES & MUJERES & & \\
\hline II CICLO & 9 & 18 & 27 & $100 \%$ \\
GRUPO 1 & & & & \\
\hline II CICL & 10 & 12 & 22 & $100 \%$ \\
GRUPO 2 & & & & \\
\hline TOTAL & & 100 &
\end{tabular}

Nota. Los datos fueron proporcionados por la oficina de registros académicos de Universidad Nacional de Frontera y adaptados a la tabla por los investigadores, elaborado por Vásquez \& Chunga (2020).

\section{Resultados}

En esta investigación se aplicaron tres cuestionarios de 9 preguntas cada uno: a los estudiantes, jerárquicos $\mathrm{y}$ docentes quienes enseñaron el curso de arte en este semestre. A continuación, se presenta una Tabla 2 aplicada para cada caso. 
Tabla 2

Contenidos del Curso de Arte Aplicada a los Estudiantes

\begin{tabular}{lll}
\hline ITEMS & F & $\%$ \\
\hline SI & 11 & 37 \\
\hline A VECES & 6 & 20 \\
\hline NO & 13 & 43 \\
\hline Total & 30 & 100
\end{tabular}

Nota. Los datos recogidos se respaldan en la encuesta que se aplicó a los estudiantes del II ciclo del curso de arte, grupo 1. En el desarrollo de los contenidos del curso de Arte, ¿has desarrollado investigación sobre los patrimonios artísticos musicales de la localidad?, elaborado por Vásquez \& Chunga (2020).

\section{Gráfica 1}

\section{Contenidos del Curso de Arte}

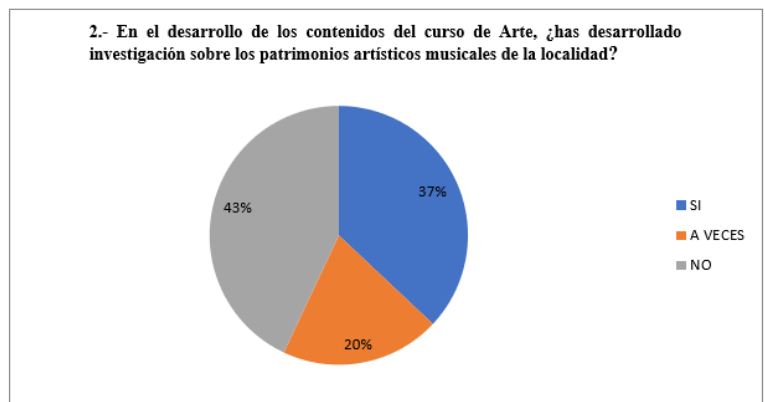

Nota. Los datos estadísticos recogidos se respaldan en la encuesta que se aplicó a los estudiantes del II ciclo del curso de arte, grupo 1, elaborado por Vásquez \& Chunga (2020).

En la Gráfica 1, aplicado a los estudiantes se muestra el $37 \%$ de encuestados ha desarrollado investigación sobre los patrimonios artísticos musicales de su localidad, el $20 \%$ lo ha realizado a veces; sin embargo, un $43 \%$, no ha realizado está investigación. Por otro lado, los resultados distan al logro de que 50\% como mínimo de estudiantes conozcan el patrimonio musical de su localidad.

En los resultados se visualiza que existe porcentaje alto $(43 \%)$ en el ítem No, lo que indica que existe un número alto de estudiantes que no han desarrollado investigación de los patrimonios musicales de su localidad. Bajo estos hallazgos, se debe reflexionar cuáles son las causas por lo que los estudiantes no investigan, qué está fallando en ese proceso de enseñanza. Esto es similar a las conclusiones dadas por Palacios quien refiere que "se debe orientar al estudiante en el proceso de investigación musical, porque para ser investigador no se improvisa, ni nace de la noche a la mañana" (2005, p.29).

\section{Tabla 3}

Investigación sobre la Música de la Localidad Aplicado a los Docentes

\begin{tabular}{lll}
\hline ITEMS & $\mathbf{F}$ & $\mathbf{\%}$ \\
\hline $\mathrm{Si}$ & 0 & 0 \\
\hline No & 2 & 100 \\
\hline Total general & $\mathbf{2}$ & $\mathbf{1 0 0}$ \\
\hline
\end{tabular}

Nota. Los datos recogidos se respaldan en la encuesta que se aplicó a las 2 docentes que enseñaron el curso de arte a los estudiantes del II ciclo del curso de arte, grupo 1 y 2. Durante el desarrollo del curso de Arte ¿los estudiantes del II ciclo han realizado investigaciones sobre la música de su localidad?, elaborado por Vásquez y Chunga (2020).

\section{Gráfica 2}

Investigación sobre la Música de la Localidad Aplicado a los Docentes

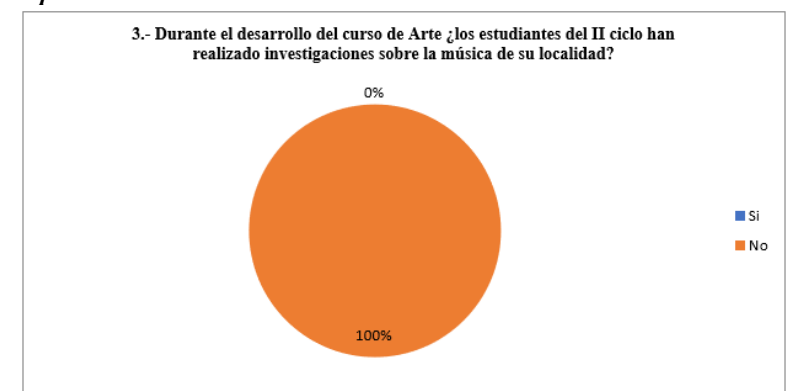

Nota. Los datos estadísticos recogidos se respaldan en la encuesta que se aplicó a los 2 docentes que enseñaron el curso de arte a los estudiantes del II ciclo del curso de arte, grupo 1 y 2 , elaborado por Vásquez y Chunga (2020).

En los resultados de la Tabla 3 y Gráfica 2, aplicada a los docentes, ambos que representan el 100\% no realizan investigación con sus estudiantes sobre música de su localidad. A su vez, se visualiza que los resultados alcanzados son desalentadores, lo cual no contribuyen en el desarrollo de la investigación artística de la localidad y por ende no genera capacidades investigativas en los estudiantes. También, se denota que los docentes no planifican sus programas curriculares con enfoque contextualizado. Frente a ello, Frega (2001) enfatiza la 
necesidad imperiosa de desenvolver este conocimiento entre los formadores de formadores musicales, es decir enseñarles todas las herramientas metodológicas para que las conozcan y luego compartan con sus estudiantes. Mientras tanto Ángeles (2017) considera que existe una relación directa entre la programación curricular y el desarrollo de competencias investigativas, algo semejante describe Mallarino al pensar:

La educación como un privilegio para el desarrollo del hombre como actor principal que apalanca los cambios de orden que se originan en la toma de decisiones diferentes a las tradicionales para los diseños curriculares, que dan lugar a criterios que rompen con la rígida separación entre las disciplinas, con la simple transmisión del conocimiento (2017, p. 02).

\section{Tabla 4}

Incorporación de la Investigación Artística en un Programa Curricular

\begin{tabular}{lll}
\hline ITEMS & F & \% \\
\hline $\mathrm{Si}$ & 4 & 100 \\
\hline No & 0 & 0 \\
\hline Total & $\mathbf{4}$ & $\mathbf{1 0 0}$ \\
\hline
\end{tabular}

Nota. Los datos recogidos se respaldan en la encuesta que se aplicó a 4 jerárquicos de la Facultad de Administración, Hotelera y de Turismo, de la Universidad Nacional de Frontera. ¿Considera usted que una propuesta de programa curricular basado en un enfoque contextualizado debe incluir la investigación artística musical?, elaborado por Vásquez \& Chunga (2020).

\section{Gráfica 3}

Incorporación de la Investigación Artística en un Programa Curricular

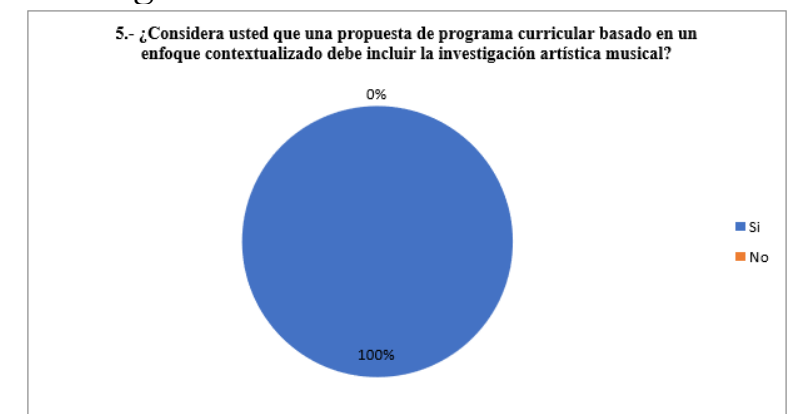

Nota. Los datos recogidos se respaldan en la encuesta que se aplicó a 4 jerárquicos de la facultad de administración, hotelera y de turismo, de la Universidad Nacional de Frontera, elaborado por Vásquez \& Chunga (2020)

Se visualiza en la Gráfica 3 que, de las personas encuestadas acerca de la importancia de considerar una propuesta de programa curricular basado en un enfoque contextualizado, $\mathrm{y}$ así ser incluido en investigación formativa artística musical, todos estuvieron de acuerdo con esta opción afirmativa, demostrando su relevancia de este, no solo en las carreras de ciencias, sino en las de humanidades como es el cado de Administración, Hotelera y de turismo, curso de arte. Esto evidencia, que los jerárquicos le dan importancia en un margen del $100 \%$ a la investigación artística en ese contexto. Igualmente, es mencionado por Capistran cuando refiere que "las autoridades educativas tienen retos que deben ser superados en materia de arte y problemáticas que deben ser atendidas" (2018, p. 1). de hecho, que van desde la atención de diseños de programas curriculares hasta su aplicación.

\section{Conclusiones}

Desde el diagnóstico del desarrollo la investigación artística musical en los estudiantes del II ciclo del curso arte se puede inferir: los docentes asignados a enseñar el curso de arte, sólo el 50\% son de especialidad, los planes curriculares sílabos no están orientados al desarrollo de investigación artística musical, no se promueven proyectos de investigación formativa relacionados a investigación artística. Se seleccionó teorías científicas, las cuales permitieron construir y validar un programa curricular contextualizado sólido que partió desde la realidad diagnóstica producto de la aplicación de instrumentos de investigación a los estudiantes, docentes y jerárquicos.

Para diseñar el programa curricular articulado de manera coherente con las competencias, objetivos, contenidos, criterios de evaluación, metodología y evaluación que desarrolle la investigación artística musical en los estudiantes del II ciclo del curso arte, se analizaron fundamentos pedagógicos, sociológicos. Como resultado, se obtuvo el programa validado donde el enfoque contextualizado recogió los aportes pedagógicos como herramienta que orienta los procesos cognitivos de enseñanza-aprendizaje y los sociológicos para interceptar el sistema 
educativo acorde a las necesidades que tiene la sociedad actual. En ese sentido, para dar un mejor soporte teórico al enfoque contextualizado, se analizó los 3 tipos de contextos que refiere Mallarino "el de intervención pedagógica horizonte de sentido, el contexto de intervención didáctica-horizonte operativo y el de contexto de intervención discursivo-horizonte epistemológico" (2017, p. 37); optándose por utilizar el de intervención pedagógica.

Se precisa que este programa curricular contextualizado contiene objetivos que facilitan los logros de aprendizaje, establecen capacidades de logros y contenidos de los saberes culturales descontextualizados (Cuellar, 2015, p. 15). De igual manera lo referido por Bocanegra "estrategias para evaluar en democracia, instrumentos de coevaluación y autoevaluación" (2007, p. 2). Por consiguiente, "la metodología facilita logros de aprendizaje y orienta el proceso de enseñanza - aprendizaje" (Del Sol et al., 2017).

Por ello, se logró que el programa curricular, contenga: objetivos, perfil de egreso, fundamentos, niveles de concreción curricular, matriz de los componentes, contenidos de planificación curricular, 8 sesiones pedagógicas con su respectivo material de trabajo y rúbricas de evaluación. Finalmente, se validó recibiendo opinión favorable de tres expertos, un licenciado en turismo, un docente de arte, un especialista en cultura para diseñar los componentes de la programación curricular.

\section{Reconocimiento}

Un agradecimiento especial a los estudiantes del II ciclo del curso de arte quienes demostraron responsabilidad, espíritu de colaboración en todo el proceso de investigación.

\section{Referencias}

Ángeles, T. J. (2017). Plan curricular y las competencias investigativas en la maestría de la Unidad de Posgrado en la Facultad de Educación de la UNMSM2017 [tesis para optar el grado académico de magíster en educación con mención en docencia en el nivel superior]. https://cybertesis.unmsm.edu.pe/bitstream/handle/20. $500.12672 / 11100 /$ Angles tj.pdf?sequence $=1 \&$ isAllo wed $=\mathrm{y}$

Bocanegra, V.B. (2007). Co-Auto evaluación. Aportes para un modelo de evaluación. Universidad Nacional Pedro Ruíz. Ediciones Odar. EIRL.

Castillo, L.S \& Herrera, V.W. (2017). Investigación Educativa. Universidad Pedro Ruíz Gallo.

Cuellar, S.M. (2015). Programación curricular de aula desde el modelo curricular sociocognitivo humanista en una institución educativa de Abancay. Repositorio de la Universidad Católica del Perú. 2017. [tesis para optar el grado de maestría con mención en currículo]. http://tesis.pucp.edu.pe/repositorio/bitstream/hand le/20.500.12404/6735/Cuellar_Laupa_Sara_Progr amacion.pdf?sequence $=1 \&$ isAllowed $=\mathrm{y}$

Del Sol, F., Tejeda, C. \& Mirabal, M. (2017). Los métodos teóricos: una necesidad de conocimiento en la investigación científico-pedagógica. Revista Edumecentro, $\quad 9 \quad$ (4) http://scielo.sld.cu/scielo.php?script=sci_arttext\&pid $=$ S2077-28742017000400021

Frega, A.L. (2001). Material visual de la Universidad Autónoma de México. Cuadernos interamericanos en educación musical. 1,1,3 5-47. http://www.revistas.unam.mx/index.php/cem/article/v iew/7306

Hurtado, J. (2000). Metodología para la Investigación Guía para la comprensión holística de la ciencia. Ediciones Quirón.

Ley Universitaria $\mathrm{N}^{\circ} 30220$ (2014). Material visual del Ministerio de Educación. http://www.minedu.gob.pe/reformauniversitaria/pdf/ley_universitaria.pdf

López-Cano, R. (2013). La investigación artística musical. Editorial Esmuc.

Mallarino, F. C. (2017). La contextualización del currículo Cognición y no verbalidad. Revista cientifica Guillermo de Ockham. 5(1), 73-80. https://www.redalyc.org/pdf/1053/105316864006.pdf

Montero, J.C. (2001). Material visual del Instituto Nacional de Musicología.

https://www.lanacion.com.ar/espectaculos/musica/ava nces-para-las-investigaciones-en-la-musicologiadocumental-nid323352/

Otaola González, P. (2015). La investigación en musicología desde una perspectiva pluridisciplinar. DEDiCA Revista de Educação e Humanidades, 77-95

Palacios, S. J. (2005). La Universidad y la Investigación Musical: de la Teoría a la Praxis. Revista Interuniversitaria de Formación del profesorado. Universidad de Zaragoza. España. V. (19) 123-156. https://www.redalyc.org/pdf/274/27419108.pdf 
Hernández-Sampieri, R., Fernández-Collado, C. \& BaptistaLucio, N. (2014). Metodología de la Investigación. 6ta edición. McGraw-Hill / Interamericana Editores, S.A.

Vegas, C. (1931). Gabinete de Musicología Indigena en el Museo de Historia Natural. https://www.segib.org/wpcontent/uploads/memorias_I_coloquio.pdf 